ISSN 1112-9867

Available online at

http://www.jfas.info

\title{
MOLECULAR STUDY OF THEILERIA CAMELENSIS AND THEILERIA DROMEDARII STRAINS BASED ON SEQUENCE OF 18 S RIBOSOMAL DNA FRAGMENT IN CAMELS
}

\author{
V. Moezi ${ }^{2}$, A. Sarani ${ }^{1, *}$, H. Hashemi ${ }^{1}$ and M. Rasekh ${ }^{1}$ \\ ${ }^{1}$ Department of clinical science, faculty of veterinary medicine, university of Zabol, Zabol, \\ Iran \\ ${ }^{2}$ Graduated from the faculty of veterinary medicine, university of Zabol, Zabol, Iran
}

Published online: 05 June 2016

\begin{abstract}
The breeding of camels in the world is growing expansionary due to increasing in consumption rate of its meat, milk and wool. Besides, The rise of profitability of this industry and importing of camels into the country through the South East borders make it important to recognize infectious diseases in this species. Theileriosis a fatal protozoan infection with worldwide distribution into the domestic animals. It has two important species, Theileria camelensis, and Theileria Dromedarii. Because of low reliability in the current visual detection of this diseases we designed this study to investigate the prevalence of camels Theileria in Zabol and Zahedan district.

Blood of 310 tick infected camels were collected from the jugular vein during July 2014 to July 2005. DNA was extracted from a whole blood sample, and PCR determined the presence of the parasite.The results showed that none of the blood contains the DNA of Theileri Camelensis and Theileria Dromedarii This result suggests that this population of camels in the southeast of country is clean from the Theileri Camelensis and Theileria Dromedarii.
\end{abstract}

Keywords: Theileri Camelensis; Theileria Dromedarii; PCR; Camelus dromedarius.

\footnotetext{
Author Correspondence, e-mail: V_Moezi@mail.com

doi: $\underline{\text { http://dx.doi.org/10.4314/jfas.8vi2s.55 }}$
} 


\section{INTRODUCTION}

The thileriosis is an important worldwide parasitic disease in cattle caused by the parasite Theileria (an obligate intracellular blood parasite) and transmitted by ticks. Theileriosis is transmitted by the tick species Hyaloma dromedarii in camels, its main host, but it is also found on the skin of cattle, sheep, goats, and donkeys (5). Yakimov et al. first reported it in camels in Russia in 1917. So far, two species of Theileria have been reported in the world: T. camelensis and T. dromedarii (1). As there is insufficient information on the microschizont stage of this parasite, and since it has been observed only its piroplasm stage in erythrocytes, its classification is not clear yet (2).

So far, no clinical symptoms have been reported for $\mathrm{T}$. dromedarii, but $\mathrm{T}$. camelensis causes a chronic disease in camels (9) with symptoms such as fever, lack of appetite, swelling of surface lymph nodes, Lacrimation, hemolytic anemia, abortion, and infertility (8). Since it is a chronic disease, and the symptoms caused in camels are mild, few reports have been published on its prevalence in the world. Its prevalence rates in Saudi Arabia, Egypt, Jordan, and Iran are 38.73, 30.4, and $15.79 \%$, respectively (7, 8, 9, and 10).

Morphological, serological, and molecular methods are used for identification of Theileria parasites. The various molecular methods researchers have used to identify species of Theileria include sequencing, PCR, and RFLP. The PCR method enjoys high sensitivity in the identification and differentiation of Theileria species, and can detect theileriosis in its earliest stages of development (3). Sequencing the 18Sr DNA gene is one of the best molecular methods for determining the strains of this parasite, and many researchers have used it worldwide.

The warm and arid climate of the Sistan and Baluchestan Province, the abundance of the tick vectors, and the extensive legal and illegal import of stock from neighboring countries that lack veterinary-sanitary standards can inflict severe economic losses on camel owners in Iran. Therefore, this research was conducted because of the insufficiency of the available information on the degree of prevalence of this disease among the camel population of the Sistan and Baluchestan Province.

\section{MATERIALS AND METHODS}

The camels used in this research were randomly selected from herds that were not vaccinated or sprayed, and the symptoms and features of the studied camels including age, gender, species, presence or absence of ticks on their bodies, and other clinical symptoms were 
recorded in the questionnaires. Blood samples were then taken from 310 camels in areas on the outskirts of Zahedan and Zabol where they were raised, and from camels imported into the province from the Mirjaveh border town, in the summer of 2014 and spring and summer of 2015. Five cc of blood were taken from the jugular vein of each camel, the samples were poured into test tubes containing anticoagulants, and commercial kits manufactured by the MBST Company were used for DNA extraction. To make sure of the accuracy of DNA extraction, some of the samples. The quality of extracted DNAs was confirmed by the agarose gel electrophoresis.

Reference primers were used for amplification and proliferation of the desired $18 \mathrm{~S}$ ribosomal DNA segment (11) (Table 1).

Table 1. Designed primers for proliferation of the hypervariable region in the 18 Sr DNA gene in the Theileria parasite

\begin{tabular}{lrr}
\hline primer & Accession number in Genbank & Primer sequence \\
\hline Forward & Hyper variable region V4 of 18 s r & 5'CACAGGGAGGTAGTGACAA \\
& DNA (Schnittger et al.2004) & G 3' \\
\hline Reverse & AJ006446 NCBI & $'$
\end{tabular}

5'AAGAATTTCACCTCTGACAG

Three 1 of DNA from each sample, 0.51 of each primer, 101 of Master Mix, and 61 of sterilized distilled water were used to make up a final volume of $20 \quad 1$ to perform the PCR. The following program was used for proliferating DNA strands employing a thermocycler (Eppendorf, Germany). The initialization step was carried out at $95^{\circ} \mathrm{C}$ for 5 minutes and consisted of 35 cycles including the denaturation stage at $94^{\circ} \mathrm{C}$ for 45 seconds, the annealing stage at $56^{\circ} \mathrm{C}$ for 45 seconds, the extension stage at $72^{\circ} \mathrm{C}$ for 45 seconds, and the final elongation stage at $72^{\circ} \mathrm{C}$ for 10 minutes. The whole reaction in this part took one hour and 48 minutes. The results of the reactions were analyzed by 81 of the PCR products on $2 \%$ agarose gel Electrophoresis. After staining the gel with ethidium bromide, it was put in the ultraviolet radiation emitting equipment so that the desired bands could be observed when ultraviolet light was emitted. 


\section{RESULTS AND DISCUSSION}

Three hundred and ten blood samples were studied and none of them contained T. camelensis or T. dromedarii. Despite the severe tick infestation, especially in imported stock, no parasites were detected. Although microscopic inspections indicated the presence of this parasite in one sample, this could not be confirmed in the molecular investigation.

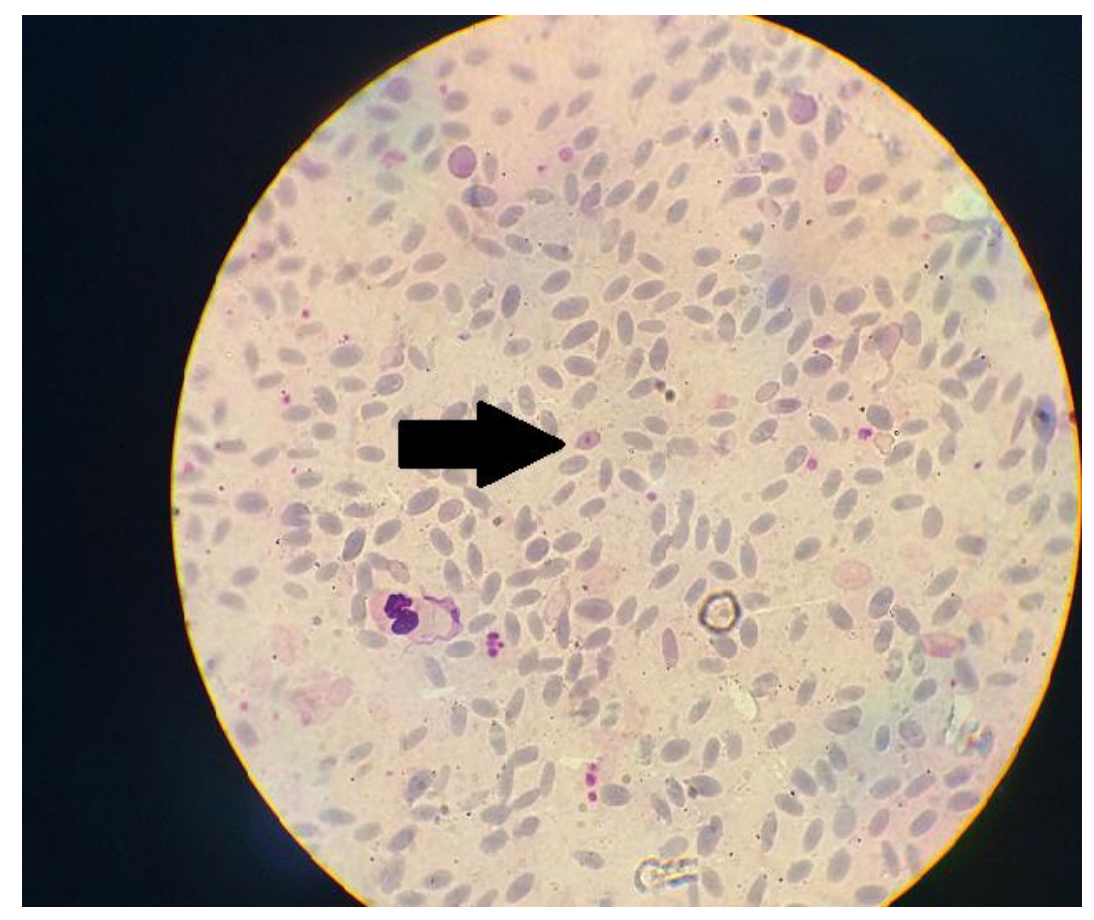

Fig.1. Theileria parasite inside a red blood cell 


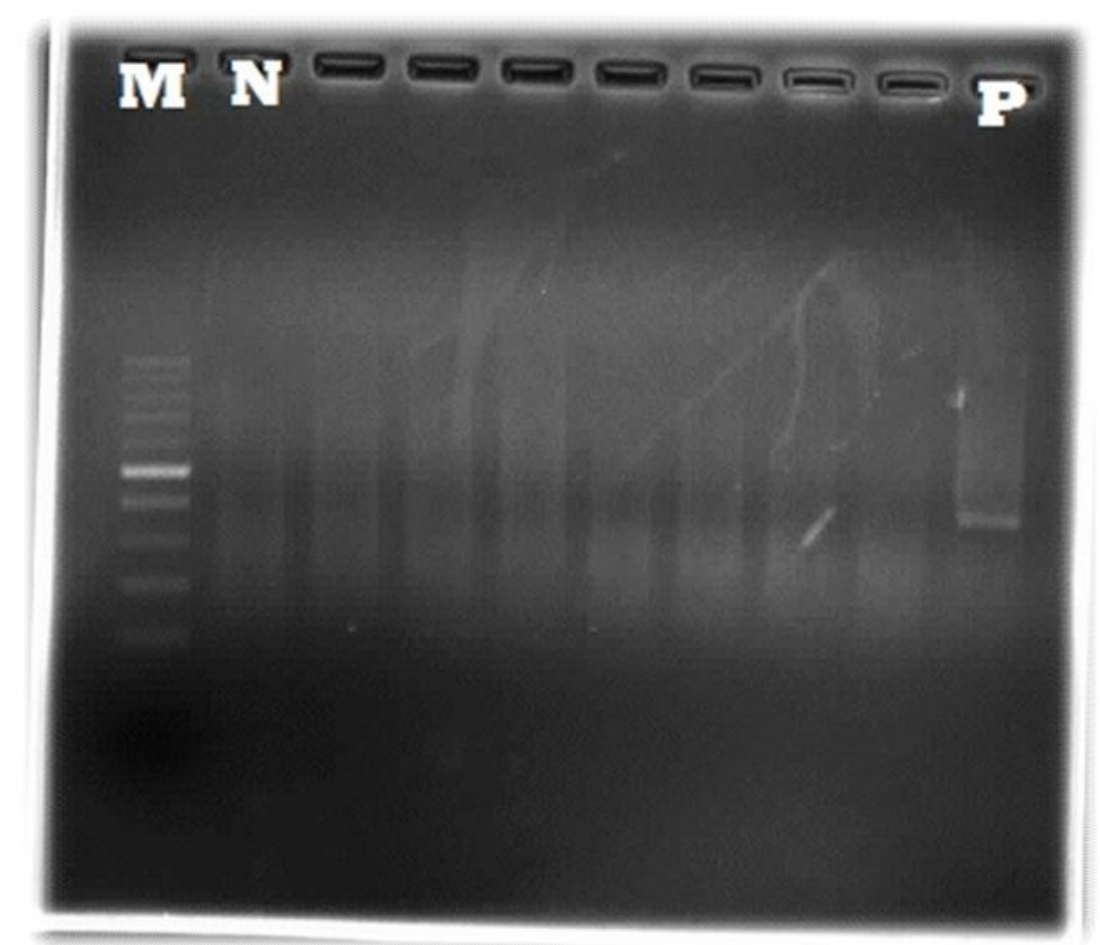

Fig.2. Results obtained from PCR products on agarose gel (M strands for marker, $\mathrm{N}$ for negative control, and $\mathrm{P}$ for positive control)

Clinical examinations of the camels revealed no distinctive symptoms in most of them (Table 2).

Table 2. Table showing the number, gender, and age groups of the studied camels

\begin{tabular}{rrrrrrr}
\hline \multicolumn{2}{r}{ camels } & & & & Age & \\
\cline { 1 - 5 } negative test & & $>2$ & $2-5$ & $5-10$ & $>10$ & Total \\
& & years & years & years & years & \\
\hline & NO & 28 & 150 & 104 & 28 &
\end{tabular}

\begin{tabular}{rlrrrrr}
\hline \multirow{2}{*}{ Gender } & Male & 20 & 120 & 55 & 12 & 310 \\
\cline { 2 - 6 } & Female & 8 & 30 & 49 & 16 & \\
\hline \multirow{2}{*}{ Present } & & 9.04 & 48.39 & 33.55 & $\% 9.04$ & $\% 100$ \\
& & $\%$ & $\%$ & $\%$ & & \\
\hline
\end{tabular}




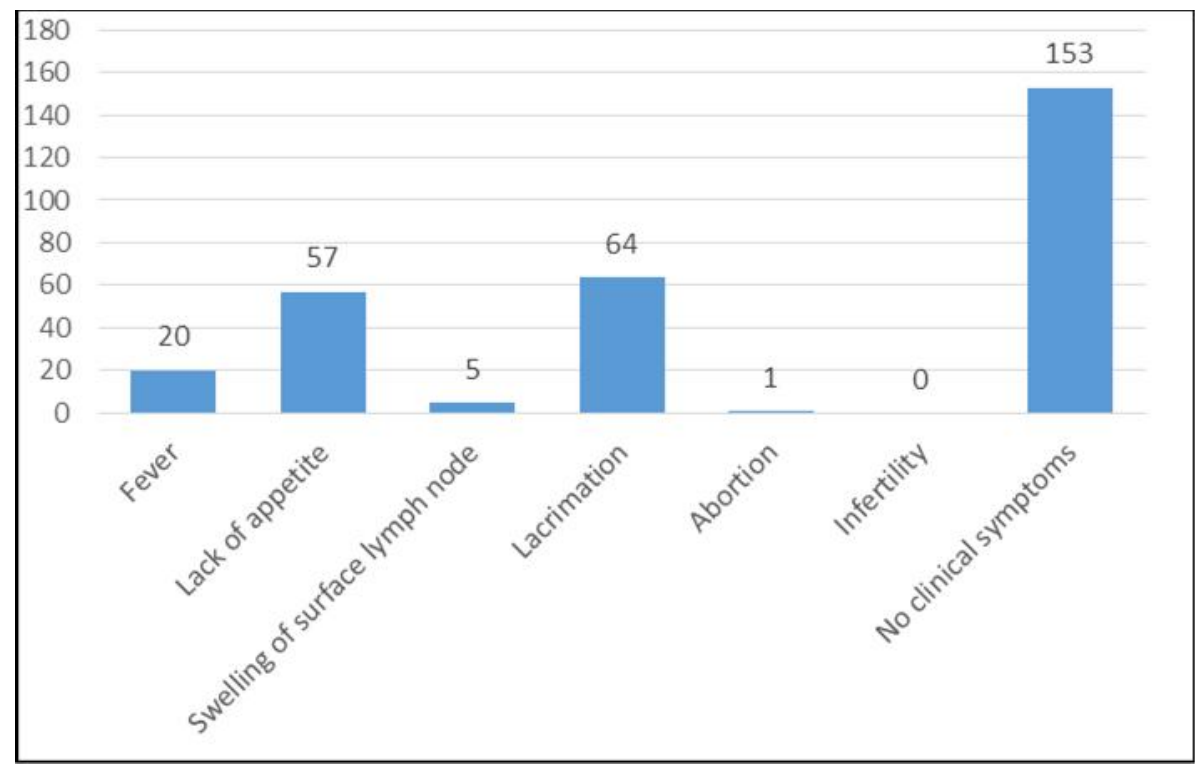

Fig.3. Results obtained from clinical examinations of the studied camels

Various studies were conducted to detect these blood parasites in the camels in Iran, and most of them used microscopic inspection and staining of blood smears. The method of using Giemsa staining and microscopic inspection does not have a high confidence coefficient since various piroplasmic stages may be observed in blood smears, and because there is the likelihood of human error. Moreover, since in endemic regions Hyaloma dromedarii ticks may be found on the bodies of other stock including cattle, detection of some theilerial bodies in blood smears of camels indicates the presence of a parasite of other stock origin with camels as its aberrant hosts. Therefore, identification of theilerial bodies requires the development of more accurate molecular methods (12).

No reports have been published so far on pathogenicity of $\mathrm{T}$. dromedarius, but $\mathrm{T}$. camelensis causes a chronic illness in camels (9) with symptoms such as fever, lack of appetite, swelling of surface lymph nodes, watery eyes, hemolytic anemia, abortion, and infertility (8). In the present research, these symptoms were not observed in the studied camels, which can suggest the low prevalence of these blood parasites among the camels in the province.

In their visual physical examination of two camels, Hamidinejat et al. noticed, and reported for the first time in Iran, the presence of Theileria parasites in red blood cells of the camels that exhibited the clinical symptoms of pale mucous membranes and skin nodules (1). In the study carried out by Borji et al. in 2009, none of the 262 samples taken from the camels transferred from Sistan and Baluchestan Province to Khorasan Razavi Province contained these blood parasites 94). In their study conducted in Yazd, Hekmatimoghaddam et al. reported the prevalence rate of these parasites in camels transferred from Yazd Province was 
$15.79 \%$ (7). Results of the present research did not indicate the presence of these blood parasites in camels, and they are in complete agreement with those found in research Borji et al. conducted. Considering the specific primers used in this study and the high sensitivity of the employed molecular method, it seems these parasites are of a very low prevalence rate among the camels in Iran. The levels of infection with diseases transmitted by tick bites depend on various factors such as genetic diversity, race, resistance of stock and presence of disease reservoir (6), and this may apply to the present study too. In addition to the above factors, there are factors including the immunity status of the stock, different sensitivities of the various races of the stock, and presence and numbers of parasites in blood, that influence the levels of infection.

\section{REFERENCES}

[1] Borji H, Razmi G, Parandeh S. Epidemiological study on haemoparasites of dromedary in Iran. Journal of Camel Practice and Research., 2009, 16, 217-219.

[2] Elghali A and Hassan S. Drop-off rhythms and survival periods of Hyalomma dromedarii (Acari: Ixodidae) fed on camels (Camelus dromedarius) in the Sudan. Veterinary Parasitology., 2010, 170, 302-306.

[3] Ezzatpour M. The Tick Vector of Pyroplasmy Agents and Methods of Controlling It, Tehran University., 1987.

[4] Gebrekidan H, Hailu A, Kassahun A, Rohousova I, Maia c, Talmi Frank D and Warburg A. Theileria infection in domestic ruminants in northern Ethiopia. Veterinary Parasitology., 2014, 200, 31-38.

[5] Hamidinejat H, Razi Jalali M, Noori M. Report on Clinical Theileriosis in a One-year Old Camel in Khuzestan Province, the Fifteenth Iranian Veterinary Congress., 2008.

[6] Hekmatimoghaddam H, Sazmand A, Rasooli A, Hamidinejat H and Jafari H. Laboratory tests in dromedary camels naturally infected with piroplasms in Iran study and review of literature. Journal of Camel Practice and Research., 2012, 19, 217-221.

[7] Ismael A, Swelum A, Khalaf A and Abouheif M. Clinical, Haematological and Biochemical Alterations Associated with an Outbreak of Theileriosis in Dromedaries (Camelus dromedarius) in Saudi Arabia. Pak Vet J., 2013, 34(2), 209-213.

[8] Nassar A. Theileria infection in camels (Camelus dromedarius) in Egypt. Veterinary Parasitology., 1992, 43, 147-149. 
[9] Qablan M, Sloboda M, Jirku M, Dwairi S, Sami Amr Z, Horin p, Lukes J and Modry D. Quest for the piroplasms in camels: Identification of Theileria equi and Babesia caballi in Jordanian dromedaries by PCR. Veterinary Parasitology., 2012, 186, 456-460.

[10] Shaikh N. Study of Three DNA Purification Methods for Use in the PCR Technique, the Journal of Hamadan University of Medical Sciences., 2004, 12(2), 10-14.

[11] Shayan P and Rahbari S. Simultaneous differentiation between Theileria spp. and Babesia spp. on stained blood smear using PCR. Parasitology Research., 2005, 97(4), 281-289.

[12] Wenyon A. 1926. A manual for medical men. Protozoology., 1926, 11.

\section{How to cite this article:}

Moezi V, Sarani A, Hashemi H and Rasekh M. Molecular study of Theileria Camelensis and Theileria dromedarii strains based on sequence of $18 \mathrm{~s}$ ribosomal DNA fragment in camels. J. Fundam. Appl. Sci., 2016, 8(2S), 399-406. 